\title{
Common mutations and polymorphisms predicting adverse cardiovascular events: current view
}

\section{"A large 'hidden heritability' observed in the genetic studies of cardiovascular adverse events is of unknown nature and may be explained by the lack of identification of the causal variants."}

\section{KEYWORDS: adverse cardiovascular events " genetics " prediction}

Cardiovascular diseases (CVDs) are a major public health concern ranked by WHO as the number one cause of death globally. Adverse cardiovascular events will largely contribute to the death of almost 23.6 million people a year by 2030 [101].

CVDs are multifactorial pathologies with a significant genetic component. Tremendous breakthroughs in the identification of the genetic variants involved have been made during the last few decades, notably thanks to the technological development of high-throughput genotyping technologies, especially genome-wide association studies (GWAS) [1], as well as the candidategene studies, which have also offered interesting results.

However, for most of the variants revealed, the transition from a statistical association to a causal mechanism has proved far from straightforward. Transcriptional analyses meet difficulties in defining the transcripts likely to mediate the associations, thus blocking the translation of genetic discoveries into an improved understanding of disease biology. Moreover, the clinical usefulness of GWAS-identified CVD loci is almost negligible at this stage.

\section{Genetic variants \& adverse cardiovascular events}

Several genes and common variants have been associated with adverse cardiovascular events.

As an example of the candidate-gene approach, a recent study assessed the effect of 48 polymorphisms previously associated with myocardial infarction (MI) and coronary artery diseases (CADs) in independent studies. Three variants (C677T in the MTHFR gene, $\mathrm{C} 242 \mathrm{~T}$ in the $p 22-P H O X$ gene and $482 \mathrm{C} / \mathrm{T}$ in the $A P O C 3$ gene) were significantly associated with increased risk of adverse effects, while the genetic score calculated by these variants predicted a $25 \%$ risk of a major adverse effect per risk allele [2].

Hereafter, we present some interesting results, mainly, but not exclusively, in the CAD field of GWAS. The intergenic SNPs rs10757278 and rs 4977574 located on chromosome 9p21 have been associated with increase risk of MI and earlyonset MI [3-6]. Significant variants associated with MI and/or coronary revascularization have been found in 6q25-27. The most significant markers are rs6922269 in the MTHFD1L gene [7]; haplotypes from rs2048327 (in SLC22A3), rs3127599 (in LPAL2), and rs7767084, rs10755578 and rs3798220 (in SLC22A3) [5,8]; and rs365302 in the FNDC1 gene [9]. In addition, the SNPs rs599839 [5,7] and rs646776 in 1p13.3 $[4,10]$ have been associated with early-onset MI and risk of CAD.

Other variants associated with early-onset $\mathrm{MI}$ or MI include the intergenic SNPs rs16893526 (6q14.1) [9], rs9982601 (21q22.11), rs1746048 (10q11.21) and rs11206510 (1p32.3), as well as the intronic SNPs rs1332844 [10], rs12526453 [4] and rs9349379 (PHACTR1) [10,11], rs17465637 (MIA3), rs1122608 (SMARCA4), rs6725887 (WDR12) [4], rs17114036 [5], rs17114046 (PPAP2B) [11] and rs1412444 (LIPA) [9,10]. Erdmann and coworkers identified the association of rs9818870 in the $M R A S$ gene and rs3739998 in the KIAA1462 gene with CAD, defined by occurrence of MI [12,13]. The association between $M R A S$ gene with CAD (rs2306374) has also been confirmed by Schunkert and coworkers [5], who also identified a novel significant association between the ADAMTS7 gene (rs3825807) and CAD. The most recent study included a discovery meta-analysis of five GWAS. The SNP rs3869109 was significantly associated with risk of CAD. It is located on chromosome

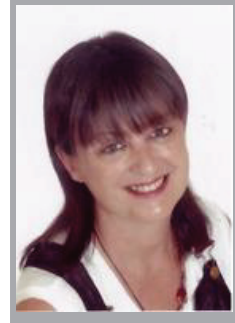

Sophie Visvikis-Siest Author for correspondence: Université de Lorraine, EA4373 'Cardiovascular Genetics', 30 Rue Lionnois, Nancy, F-54000, France Tel.: +330607602569 Fax: +330383321322 sophie.visvikis-siest@inserm.fr

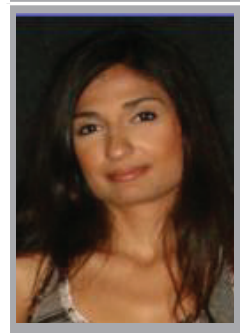

Maria G Stathopoulou

Université de Lorraine, EA4373 'Cardiovascular Genetics', 30 Rue Lionnois, Nancy, F-54000, France

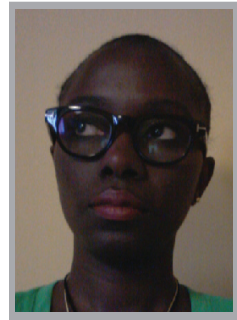

Ndeye Coumba Ndiaye Université de Lorraine, EA4373 'Cardiovascular Genetics', 30 Rue Lionnois, Nancy, F-54000, France 
$6 \mathrm{p} 21.3$ in the MHC; thus, supporting the role of inflammation and immune system in the pathogenesis of CVDs [14].

The observation that, in multiple studies, the replication of specific chromosomal regions, including the 9p21, 6q25-27 and the PHACTR1, PPAP2B, LIPA and PITX2 genes, are associated with coronary adverse events is very promising.

\section{Applications in clinical practice: achievements \& future challenge}

Many attempts have been made to develop genetic profiles using the results of the genetic studies, but their value was very limited for predicting personal risk.

For useful clinical prediction, genetic testing must be both sensitive and specific in discriminating between those who will and will not develop the disease. The standard measure of this discriminative capacity may be provided by the receiver-operating characteristic curves, which plot the performance of a given diagnostic test in terms of those two factors. In clinical settings, an area under the curve of $0.8-0.9$ is considered as a 'good' test. Genetic variants identified so far do not substantially improve the discriminative accuracy of disease prediction based on clinical characteristics.

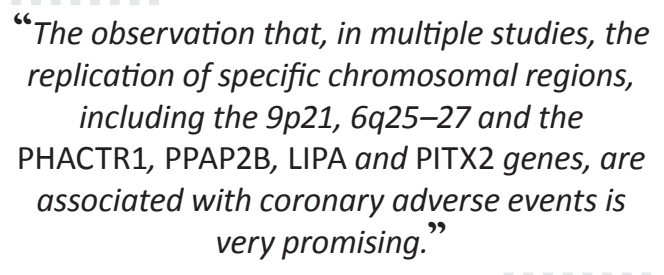

For example, when evaluating the ability of a panel of SNPs to distinguish patients with coronary heart disease, researchers noted that the area under the receiver-operating characteristic curve was low, indicating that conventional risk factors and family history are the best predictive biomarkers [15].

One fundamental problem lies in the modest effect sizes of the discovered variants, and therefore the limited proportion of heritable variance explained. For example, the 13 newly and ten loci previously identified by Schunkert and coworkers account for only $10 \%$ of additive genetic heritability of CAD [5]. Therefore, an important part of the missing heritability remains to be discovered.

A large 'hidden heritability' observed in the genetic studies of cardiovascular adverse events is of unknown nature and may be explained by the lack of identification of the causal variants.
Gene-gene $(\mathrm{G} \times \mathrm{G})$ or epistatic interactions, and both low minor allele frequencies (MAFs) and rare variants (items previously reviewed by our team [16]), may play an important role in discovering new genes [16].

$G \times G$ interactions are issues that blind many GWAS. Even if, both parametric and nonparametric multilocus methods have been developed to detect such interactions, current epistatic designs do not have genomewide coverage and their application to highdimensional genome-wide data, including all imaginable SNPs, remains a crucial challenge [16].

Furthermore, GWAS have predominantly focused on common variants (a threshold of 5\% for MAF is used in GWAS) and ignored low MAF and rare variants, which may have a greater effect, owing to methodological difficulties [16]. A great deal of hope rests on the expectation that largescale sequencing studies will reveal causal variants in the low $(0.5 \%<\mathrm{MAF}<5 \%$ in the general population) and rare (MAF $<0.5 \%$ ) frequency ranges. Moreover, structural large variants $(>500 \mathrm{~kb})$ such as deletions and duplications (copy-number variants) have been shown to affect gene expression and pathways in humans $[16,17]$. These variations are rare $(\mathrm{MAF}<0.05 \%)$ but collectively common as they they are present in approximately $8 \%$ of the total population [16].

Moreover, genetic prediction has been shown to be slightly more effective in certain groups, such as the young $[18,19]$ or those with increasing duration of follow-up [20], but at the individual level, accuracy still falls well below any reasonable threshold for clinical utility.

In general, although $\mathrm{G} \times \mathrm{G}$ interactions and causal allele discovery in the lowest MAF ranges will demand alternative discovery methods, as well as specific statistical software, populations and translational approaches, it is expected that they will have larger effect sizes than the common variants found to date, and will therefore provide valuable boosts to predictive performance.

Even if individual prediction is not yet feasible, risk allele scores have the capacity to highlight groups at either end of the risk distribution. An example of the utility of genetic tests in risk stratification within groups is the increased efficiency of clinical trial design [21].

The identification of high genetic risk groups seems to be important for the design of targeted preventive public health measures, as lifestyle interventions have been shown to be beneficial in these groups [22,23]. However, the improvement of prognostic and diagnostic 
precision will result after complete identification of the predisposing genetic factors, as well as after integration of other relevant information, including environmental factors and epigenetic modifications.

Gene-environment $(G \times E)$ interaction studies could highlight the interplay between these factors and could help identify different loci effects according to modifiable risk factors. A successful $G \times E$ study should take into account sample size, exposure assessment and heterogeneity. Smith and Day proposed that, for the detection of an interaction, a sample size at least four-times greater than that required for detecting a main effect of comparable magnitude is required [24]. Although difficult, $\mathrm{G} \times \mathrm{E}$ interactions could help in understanding discrepancies due to heterogeneous exposure [16].

Children represent an attractive population for the identification of primary genetic determinants of complex polygenic traits and for $\mathrm{G} \times \mathrm{E}$ interactions investigations as they are less susceptible to chronic diseases and medication use, and less exposed to environmental factors compared with adults.

\section{"Gene-gene or epistatic interactions, and both low minor allele frequencies and rare variants ... may play an important role in discovering new genes."}

Moreover, although pathways acting through epigenetic mechanisms to modify gene expression and DNA methylation have given fruitful results in other chronic diseases, they are yet to be studied in CVDs.

Finally, gene-drug interactions have been also shown to modify risk in patients. Among the medications prescribed for CVDs, clopidogrel, a thienopyridine commonly used as antiplatelet agent, has gained significant attention owing to its interactions with genetic variants that seem to modify its responsiveness and clinical efficacy. CYP2C19 mediates the production of its active metabolite and polymorphisms of this gene have been associated with poor response to clopidogrel, lower antiplatelet activity and increased risk of cardiovascular ischemic events [25]. However, the most recent systematic review and meta-analysis suggested that CYP2C19 polymorphisms do not affect the clinical efficacy of clopidogrel in patients with ischemic CVDs [26]. Nevertheless, the study of interactions between genes and medications is of great importance in the bid to develop genetic tools oriented to personalized medicine.

\section{Conclusion \& future perspective}

The majority of the identified SNPs are rarely the causal variants but they are usually indirectly associated with the traits, owing to their close genomic proximity with the causal variant. The next step is genome annotation and identification of the causal variants directly implicated in the pathophysiology of the disease and the increased risk of adverse events. Furthermore, the identified SNPs are often located far from protein-coding genes, thus the identification of causal variants is a great challenge. The use of transcriptomics tools could give important results in the direction of identification of the functional variants in terms of gene-expression modification. Furthermore, sequencing of the regions of interest could help in identifying the causal polymorphisms or mutations, for example in cases were causal variants have obvious functional consequence, such as a nonsynonymous mutation within the coding region. Sequencing techniques could also identify polymorphisms that cannot be assessed in GWAS owing to low MAFs. Thus, the use of a combination of new technologies could eventually lead to the identification of the true disease genetic variants.

Another important issue is that a small proportion of heritability is still attributed to the identified associations. The identification and validation of variants explaining a higher percentage of the heritability of cardiovascular events will improve the effectiveness of genetic tools in the prevention and prognosis of disease and the development of personalized therapy.

Some approaches that could increase the predictive efficacy of the genetic research have been discussed. The assessment of $\mathrm{G} \times \mathrm{G}$ and $\mathrm{G} \times \mathrm{E}$ interactions is necessary, as these interactions may modify the direct genetic effects on the traits. Although the results are promising [27], these fields are not extensively assessed, mainly owing to issues of statistical power, sample size, software specificity and methodological design.

Finally, gene-drug interactions should also be further assessed. Cardiovascular events are common in nondiagnosed subjects but also in CVD patients treated with a variety of medications. For these high-risk patients, these interactions could explain a significant amount of diversity in responsiveness and efficacy of the medications, and study of this could have important applications in clinical practice.

In summary, genetic research has provided a considerable number of genetic variants that seem to be involved in adverse cardiovascular 
events. Despite important discoveries, the goal of identification of genetic markers for CVD prevention, prediction and treatment through personalized medicine is yet to be achieved.

Future research must detect low MAF and rare variants, include $\mathrm{G} \times \mathrm{G}$ and $\mathrm{G} \times \mathrm{E}$ interactions, and involve subsequent transcriptomic and proteomic investigations. The use of new technologies and the application of the experience gained by previous methodologies might further reveal the functional and mechanistic pathways involved in CVD processes, consequently allowing their use as predictive and possible therapeutic tools in the near future.

\section{Financial \& competing interests disclosure}

The authors have no relevant affliations or financial involvement with any organization or entity with a financial interest in or financial conflict with the subject matter or materials discussed in the manuscript. This includes employment, consultancies, honoraria, stock ownership or options, expert testimony, grants or patents received or pending, or royalties.

No writing assistance was utilized in the production of this manuscript.

\section{References}

1 Manolio TA. Genomewide association studies and assessment of the risk of disease. N. Engl. J. Med. 363(2), 166-176 (2010).

2 Andreassi MG, Adlerstein D, Carpeggiani C et al. Individual and summed effects of highrisk genetic polymorphisms on recurrent cardiovascular events following ischemic heart disease. Atherosclerosis 223(2), 409-415 (2012).

3 Helgadottir A, Thorleifsson G, Manolescu A et al. A common variant on chromosome $9 \mathrm{p} 21$ affects the risk of myocardial infarction. Science 316(5830), 1491-1493 (2007).

4 Kathiresan S, Voight BF, Purcell S et al. Genome-wide association of early-onset myocardial infarction with single nucleotide polymorphisms and copy number variants. Nat. Genet. 41(3), 334-341 (2009).

5 Schunkert H, Konig IR, Kathiresan S et al. Large-scale association analysis identifies 13 new susceptibility loci for coronary artery disease. Nat. Genet. 43(4), 333-338 (2011).

6 Takeuchi F, Yokota M, Yamamoto K et al. Genome-wide association study of coronary artery disease in the Japanese. Eur. J. Hum. Genet. 20(3), 333-340 (2011).

7 Samani NJ, Erdmann J, Hall As et al. Genomewide association analysis of coronary artery disease. N. Engl. J. Med. 357(5), 443-453 (2007).

8 Tregouet DA, Konig IR, Erdmann J et al. Genome-wide haplotype association study identifies the SLC22A3-LPAL2-LPA gene cluster as a risk locus for coronary artery disease. Nat. Genet. 41(3), 283-285 (2009).

9 Wild PS, Zeller T, Schillert A et al. A genomewide association study identifies $L I P A$ as a susceptibility gene for coronary artery disease. Circ. Cardiovasc. Genet. 4(4), 403-412 (2011).

10 Peden JF, Hopewell JC, Saleheen D et al. Coronary Artery Disease (C4D) Genetics Consortium. A genome-wide association study in Europeans and South Asians identifies five new loci for coronary artery disease. Nat. Genet. 43(4), 339-344 (2011).

11 Mehta NN. A genome-wide association study in Europeans and South Asians identifies 5 new loci for coronary artery disease. Circ. Cardiovasc. Genet. 4(4), 465-466 (2011).

12 Erdmann J, Grosshennig A, Braund PS et al. New susceptibility locus for coronary artery disease on chromosome 3q22.3. Nat. Genet. 41(3), 280-282 (2009).

13 Erdmann J, Willenborg C, Nahrstaedt J et al. Genome-wide association study identifies a new locus for coronary artery disease on chromosome 10p11.23. Eur. Heart J. 32(2), 158-168 (2010).

14 Davies RW, Wells GA, Stewart AF et al. A genome-wide association study for coronary artery disease identifies a novel susceptibility locus in the major histocompatibility complex. Circ. Cardiovasc. Genet. 5(2), 217-225 (2012).

15 Jeemon P, Pettigrew K, Sainsbury C, Prabhakaran D, Padmanabhan S. Implications of discoveries from genome-wide association studies in current cardiovascular practice. World J. Cardiol. 3(7), 230-247 (2011).

16 Ndiaye NC, Azimi Nehzad M, El Shamieh S, Stathopoulou MG, Visvikis-Siest S.

Cardiovascular diseases and genome-wide association studies. Clin. Chim. Acta 412(19-20), 1697-1701 (2011).

17 Henrichsen CN, Vinckenbosch N, Zollner S et al. Segmental copy number variation shapes tissue transcriptomes. Nat. Genet. 41(4), 424-429 (2009).

18 De Miguel-Yanes JM, Shrader P, Pencina MJ et al. Genetic risk reclassification for Type 2 diabetes by age below or above 50 years using 40 Type 2 diabetes risk single nucleotide polymorphisms. Diabetes Care 34(1), 121-125 (2011).

19 Meigs JB, Shrader P, Sullivan LM et al. Genotype score in addition to common risk factors for prediction of Type 2 diabetes. N. Engl. J. Med. 359(21), 2208-2219 (2008).
20 Lyssenko V, Jonsson A, Almgren P et al. Clinical risk factors, DNA variants, and the development of Type 2 diabetes. N. Engl. J. Med. 359(21), 2220-2232 (2008).

21 Schork NJ, Topol EJ. Genotype-based risk and pharmacogenetic sampling in clinical trials. J. Biopharm. Stat. 20(2), 315-333 (2010).

22 Florez JC, Jablonski KA, Bayley $\mathrm{N}$ et al. TCF7L2 polymorphisms and progression to diabetes in the Diabetes Prevention Program. N. Engl. J. Med. 355(3), 241-250 (2006).

23 Hivert MF, Jablonski KA, Perreault L et al. Updated genetic score based on 34 confirmed Type 2 diabetes loci is associated with diabetes incidence and regression to normoglycemia in the diabetes prevention program. Diabetes 60(4), 1340-1348 (2011).

24 Smith PG, Day NE. The design of case-control studies: the influence of confounding and interaction effects. Int. J. Epidemiol. 13(3), 356-365 (1984).

25 Shuldiner AR, O'Connell JR, Bliden KP et al. Association of cytochrome P450 2C19 genotype with the antiplatelet effect and clinical efficacy of clopidogrel therapy. JAMA 302(8), 849-857 (2009).

26 Bauer T, Bouman HJ, Van Werkum JW, Ford NF, Ten Berg JM, Taubert D. Impact of CYP2C19 variant genotypes on clinical efficacy of antiplatelet treatment with clopidogrel: systematic review and metaanalysis. BMJ343, d4588 (2011).

27 El Shamieh S, Ndiaye NC, Stathopoulou MG et al. Functional epistatic interaction between rs6046G $>\mathrm{A}$ in $F 7$ and rs5355C $>\mathrm{T}$ in $S E L E$ modifies systolic blood pressure levels. PloS One 7(7), e40777 (2012).

\section{Website}

101 WHO. Cardiovascular diseases. www.who.int/mediacentre/factsheets/fs317/ en/index.html 